\title{
Atmospheric Effects for the Ground-Based Calibration of Orbital UV Telescopes
}

\author{
G. Chiritoi*, E. M. Popescu, A. A. Radu, A. Caramete \\ Institute of Space Science, Magurele, Romania \\ E-mail: gabriel.chiritoiespacescience.ro \\ for the JEM-EUSO Collaboration
}

\begin{abstract}
One of the major issues in the detection of the UV yield of EASs by orbital UV telescopes is the optical calibration of the focal surface detector, which in turn requires advanced knowledge of the atmosphere in the FoV of the telescope. As such, we report here on the evaluation of the GLS as a ground-based optical system for the in-orbit calibration of instruments such as the Mini-EUSO and JEM-EUSO UV telescopes. Our study is done using GBSatCal (Ground-Based Satellite Calibration) software package, which has been designed specifically for this task. This package allows us to consider different types of radiation sources (e.g. lasers, Xe-flashers and high-power UV LEDs), of focal surface detector geometries and of atmospheric models (e.g. USAS 1976 and NRLMSISE-00), as well as to determine the optimal calibration geometry.
\end{abstract}

The 34th International Cosmic Ray Conference,

30 July- 6 August, 2015

The Hague, The Netherlands

${ }^{*}$ Speaker. 


\section{Introduction}

The detection of the UV fluorescence yield of Extended Air Showers (EAS) produced by UltraHigh Energy Cosmic Rays (UHECR) by ground observatories (e.g. the Pierre Auger Observatory (PAO) [1] and the Telescope Array (TA) [2]) is a fairly common EAS study technique. However, one of its major drawbacks is the high sensitivity to atmospheric conditions, which imposes tight requirements on the local atmospheric monitoring and on the detectors calibration schedule. For this reason, both ground observatories above have dedicated Atmospheric Monitoring Systems (AMSs) for the former purpose, consisting of LIDARs and infrared (IR) cloud monitoring cameras, and Central Laser Facilities (CLFs) consisting Xe-flashers and steerable lasers for the latter [3], [4].

Similar considerations apply, of course, to orbital observatories such as JEM-EUSO [5], MiniEUSO [6], TUS [7] and KLYPVE [8]. Most of them also have attached dedicated AMSs, as well as light systems for the in-situ calibration of the instruments to ensure the accuracy and reliability of their data and results. Furthermore, ground-based light systems similar to those of the PAO and TA CLFs for the in-orbit instrument cross-calibration are also under consideration.

The purpose of this paper is to provide a preliminary evaluation of such ground-based light systems for the cross-calibration of orbital UV telescopes by indirect illumination. The evaluation relies on the use of two of the most common atmospheric models, namely the 1976 US Standard Atmosphere Model and the NRLMSISE-00 model of the atmosphere.

The paper is organized as follows. In Section 2 we briefly review the technical and operational characteristics of the JEM-EUSO and Mini-EUSO instruments that are relevant to the present work, as well as the characteristics of the UV background in which they operate. We continue in Section 3 with a brief description of the GBSatCal software package that was developed specifically for the study of ground-based calibration systems, and with a description of the specific atmospheric conditions used in the present work. Section 4 is dedicated to the presentation and discussion of the results of our study, and the paper concludes in Section 5 with a summary of the results and plans for future work.

\section{The JEM-EUSO and Mini-EUSO UV instruments}

Both the JEM-EUSO and Mini-EUSO instruments are essentially UV telescopes with refractive optics to be housed on the International Space Station (ISS) at an average orbital altitude of $\sim 400 \mathrm{~km}$. Mini-EUSO is a ROSCOSMOS-ASI JEM-EUSO pathfinder mission scheduled for launch in a couple of years. Its instrument, much like the name suggests, is a smaller version of the JEM-EUSO instrument which will be mounted inside the Russian "Zvezda" module of ISS on a porthole with a UV transparent window, and will have a fixed orientation at the station's nadir. By contrast, the full JEM-EUSO UV telescope will be mounted on the outside of the Japanese Module of the ISS, and will be able to operate in both nadir and tilted mode.

The mission and instrument relevant to the present work, as well as the main characteristics of the background radiation environment in which they will operate are summarized below.

a) The Mini-EUSO telescope

The Mini-EUSO telescope consists of 2 Fresnel lenses, mounted above the UV transparent porthole window, which focus the light onto a focal surface consisting of $48 \times 48$ pixels [6]. The diameter of 
its input aperture is $25 \mathrm{~cm}$, and the telescope has a full $\mathrm{FoV}$ of $28^{\circ}$. The average single pixel FoV is $\sim 0.53^{\circ}$, corresponding to a ground footprint of $\sim 6.5 \mathrm{~km}$ in size. For an average ISS orbital speed of $\sim 7.67 \mathrm{~km} / \mathrm{s}$, the average speed of the ISS nadir projection onto the Earth surface and relative to the Earth's surface is $\sim 6.94 \mathrm{~km} / \mathrm{s}$, corresponding to an average single pixel time of sight on the ground of $\sim 0.94 \mathrm{~s}$. The Gate Time Unit (GTU) of the DAQ electronics is $2.5 \mu \mathrm{s}$.

b) The JEM-EUSO telescope

The full JEM-EUSO telescope consists of 3 Fresnel lenses which focus the light onto a focal surface consisting of 315,648 pixels. The doubly side-cut input aperture has a size $\sim 4.56 \mathrm{~m}^{2}$, and the full FoV of the instrument is $\sim 60^{\circ}$ [9]. The average single pixel FoV is $\sim 0.074^{\circ}$, corresponding to a ground footprint of $\sim 560 \mathrm{~m}$, and under similar conditions as above, the average single pixel time of sight on the ground is $\sim 0.081 \mathrm{~s}$, at the same value of the DAQ GTU of $2.5 \mu \mathrm{s}$.

c) The UV background environment

As far as the UV background environment is concerned, we consider here for both instruments a background radiance of $\sim 500$ photons $/ \mathrm{ns} \cdot \mathrm{sr} \cdot \mathrm{cm}^{2}$ in the UV wavelength range $300-400 \mathrm{~nm}$, which is a rather commonly used value in JEM-EUSO numerical studies (e.g. [10]) and which is close to the average radiance corresponding to a duty cycle of $\sim 21 \%$ for the JEM-EUSO telescope [11]. This radiance value leads to detection threshold of $\sim 10$ photons/pixel for both instruments.

\section{The Simulation Software}

The numerical work in the present work has been carried out using the dedicated GroundBased Satellite Calibration (GBSatCal) software package developed at the Institute of Space Science. The package is the satellite calibration counterpart of the LaserSim package developed and used in the past for the corresponding CLF calibration of the PAO and TA fluorescence detectors. From the technical viewpoint, GBSatCal calculates the number of photons per pixel reaching the input aperture of an orbital telescope indirectly illuminated by la a ground light-source shooting a pulsed light-beam in the atmosphere from a given altitude and at a given elevation angle, as illustrated in Fig.1.

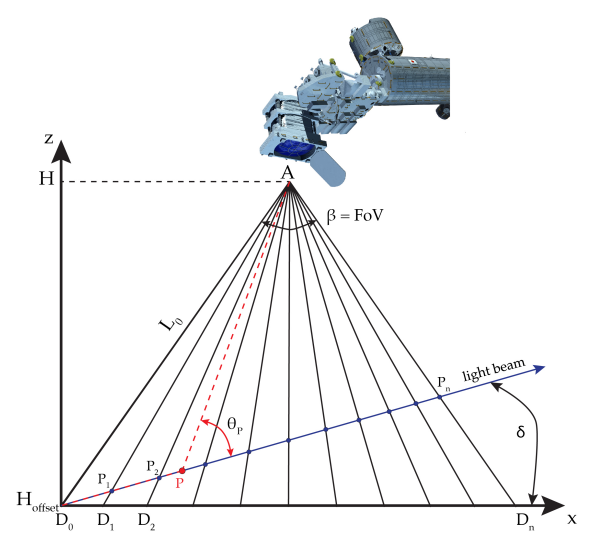

Figure 1: GBSatCal geometry setup.

The mechanism governing the indirect illumination of the telescope the wavelength dependent Rayleigh scattering, which is accounted for by the code based on atmospheric composition and 
includes molecular depolarizability effects for the relevant atmospheric species. All simulations are carried out for wavelength $\lambda=365 \mathrm{~nm}$, to ensure that light extinction is dominated by Rayleigh scattering no atmospheric absorption phenomena are present. The choice of wavelength is related to the particular light-sources that are evaluated by the present work, namely LedEngin high-power LEDs with optical output centered on this wavelength (e.g. [12], and lasers that can be tuned to it.

The major advantage of GBSatCal is that it can use different atmospheric models to calculate the amount of light transported from the source to the telescope input aperture. At present, the software can use the US 1976 Standard Atmosphere (USAS) [13] and the NRLMSISE-00 (NRLMSISE) [14] models. Since the USAS model is a mid-latitude quiescent geomagnetic model, in the present work, in order to allow for a comparison of the two models, we have used similar settings for the NRLMSISE model, namely $45^{\circ}$ for the latitude and longitude, $<$ F10.7 $>=F 10.7=150$ for the corresponding average and current 10.7 radio fluxes, and $\mathrm{A}_{p}=4$ for the $3 \mathrm{hr}$ average magnetic index. The results of our simulations are presented in the following section.

\section{Results and Discussion}

The first set of results are illustrated in Fig.2 and Fig.3. The graphs show the total number of photons per $2.5 \mu$ s pulses reaching the input aperture of the Mini-EUSO and JEM-EUSO telescopes as a function of the beam intensity at the source and for several beam diameters. The source is placed at sea-level, and the beam elevation angle is $\delta=0^{\circ}$. For brevity reasons, we have included here only the results corresponding to the NRLMSISE atmospheric model. The differences between the results obtained with the NRLMSISE and USAS models are less than $1 \%$, due to our choice for the former to represent mid-latitude quiescent geomagnetic activity conditions similar to the latter. The same holds true for all results presented in this section.

These graphs are essentially "calibration" graphs that are used to evaluate the viability of different types of light sources for indirect illumination cross-calibration purposes. The horizontal dashed lines in these graphs represent the total illumination level of the telescopes required for the first pixel (i.e. the pixel closest to the light source according to the geometry in Fig.1) to be above the 10 photon background threshold (see Section 2.c) and for the first 20 pixels (the $20^{\text {th }}$ pixel included) in the same row/column to be above the threshold.

Referring now to Mini-EUSO (Fig.2), and taking into consideration that high-power LEDs have intensities of $\sim 10^{5} \mathrm{~W} / \mathrm{m}^{2}$ and beam diameters of $\sim 10 \mathrm{~mm}$ while lasers used by the CLFs have intensities of $\sim 10^{11} \mathrm{~W} / \mathrm{m}^{2}$ and similar diameters, it is immediately clear that lasers can illuminate more than 20 consecutive pixels in a row/column, and hence they are more than appropriate for the ground-based cross-calibration of the instrument. For high-power LEDs, the situation is different. In order to indirectly illuminate just the first pixel, for the LED intensity above one would need a $200 \mathrm{~mm}$ diameter beam, i.e. a number of $\sim 400$ LEDs, which is clearly unfeasible.

For JEM-EUSO (Fig.3), the situation improves significantly. Lasers are still a feasible for ground-based instrument cross-calibration, but now one needs only $\sim 25$ high-power LEDs (e.g. a $5 \times 5$ LED source matrix) to illuminate form the ground more than 20 consecutive pixels in a row/column, which is also a viable solution. Of course, the difference between the two cases is due to the different pixel FoVs of the two instruments. 


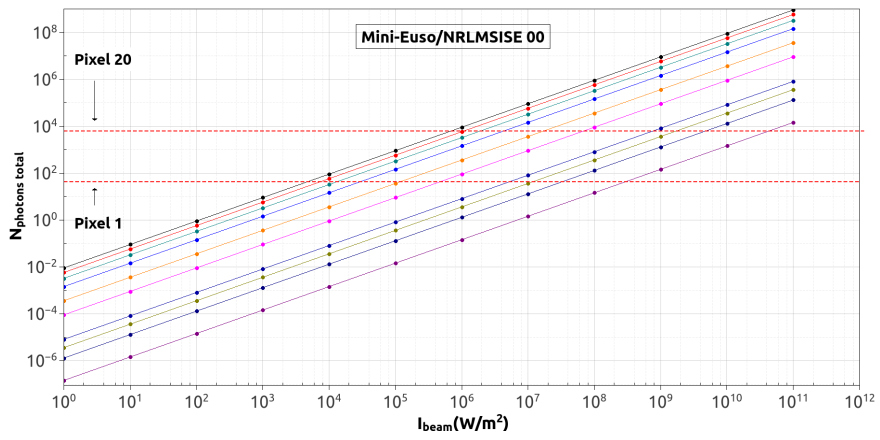

Figure 2: Total number of photons reaching the Mini-EUSO telescope input aperture as a function of the beam intensity for different diameters of the light beam. The source is placed at sea-level and the beam elevation angle is $\delta=0^{\circ}$. From bottom to top, the beam diameters are $2 \mathrm{~mm}, 6 \mathrm{~mm}, 10 \mathrm{~mm}, 15 \mathrm{~mm}, 50 \mathrm{~mm}$, $100 \mathrm{~mm}, 200 \mathrm{~mm}, 300 \mathrm{~mm}, 400 \mathrm{~mm}$ and $500 \mathrm{~mm}$.

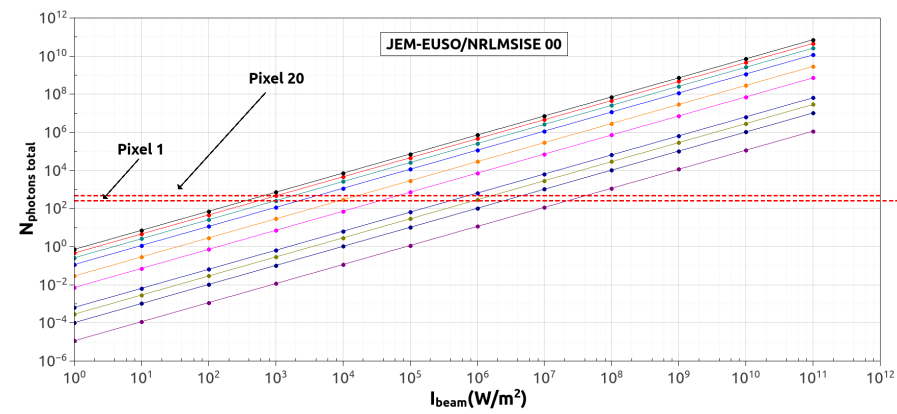

Figure 3: Total number of photons reaching the JEM-EUSO telescope input aperture as a function of the beam intensity for different diameters of the light beam. The source is placed at sea-level and the beam elevation angle is $\delta=0^{\circ}$. From bottom to top, the beam diameters are $2 \mathrm{~mm}, 6 \mathrm{~mm}, 10 \mathrm{~mm}, 15 \mathrm{~mm}, 50 \mathrm{~mm}$, $100 \mathrm{~mm}, 200 \mathrm{~mm}, 300 \mathrm{~mm}, 400 \mathrm{~mm}$ and $500 \mathrm{~mm}$.

The next results in Fig.4-Fig.7 refer to the optimization of the illumination geometry in terms of beam elevation angle and source altitude.

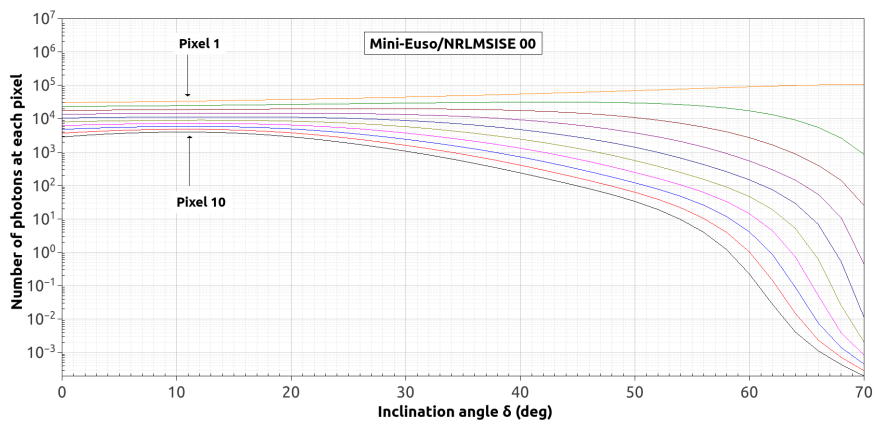

Figure 4: Variation of the number of photons reaching the input aperture of the Mini-EUSO telescope as a function of the beam elevation angle for $\mathrm{I}_{\text {beam }}=10^{11} \mathrm{~W} / \mathrm{m}^{2}$ and $6 \mathrm{~mm}$ beam diameter.

As both the lasers and the high-power LEDs exhibit similar behavior, for brevity reasons we restrict 
ourselves only to the lasers case ( $\mathrm{I}_{\text {beam }}=10^{11} \mathrm{~W} / \mathrm{m}^{2}$ and $6 \mathrm{~mm}$ beam diameter).

For both Mini-EUSO (Fig.4) and JEM-EUSO (Fig.5), it is immediately apparent that as the elevation angle $\delta$ increases, so does the number of photons reaching the pixels. Moreover, all pixels with the exception of pixel 1 (the closest to the source, according to the geometry in Fig.1), exhibit maxima in the number of received photons, and the elevation angles of these maxima decrease with increasing distance of the pixel from the source. Due to the different pixel FoVs of the two instruments, the maxima per pixels occur - generally speaking - at lower elevation angles for Mini-EUSO, and at higher elevation angles for JEM-EUSO.

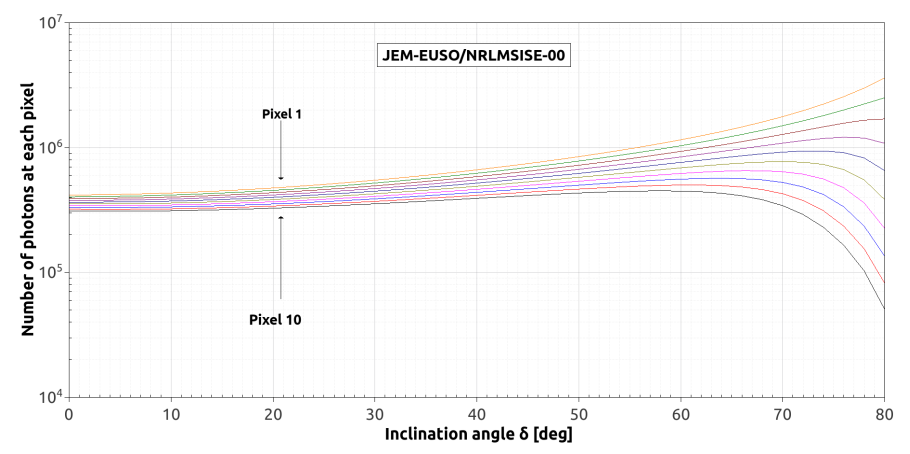

Figure 5: Variation of the number of photons reaching the input aperture of the Mini-EUSO telescope as a function of the beam elevation angle for $\mathrm{I}_{\text {beam }}=10^{11} \mathrm{~W} / \mathrm{m}^{2}$ and $6 \mathrm{~mm}$ beam diameter.

In terms of source altitude, the results in Fig. 6 and Fig. 7 for $\delta=0^{\circ}$ are quite surprising and a bit counterintuitive. As the altitude of the source increases, the number of photons reaching pixel 1 decreases, as one would expect due to the decreasing density of the atmosphere with altitude. The counterintuitive part occurs for pixels in the row that are increasingly farther away from the source. As one can see in Fig.6 and Fig.7, for both Mini-EUSO and JEM-EUSO, in the altitude range 0-10 $\mathrm{km}$, pixels in the row/column increasingly farther apart from the source receive more and more photons (up to 1000 times more for Mini-EUSO!) compared to the number of photons received for s sea-level source. As surprising as it may be, the explanation of this result is in the end quite pedestrian. Indeed, the number of photons scattered away from the beam towards each telescope pixel depends on the balance between the local photon flux in the light beam and the local density of atmospheric scatterers. As the altitude increases, fewer and fewer photons are scattered away from the beam in the FoVs of the pixels closer to the source, leaving more and more photons in the beam to be scattered in the FoVs of the pixels that are further apart form the source, and hence leading to a larger number of photons - compared to the number of photons at sea-level - reaching the pixels farther away from the source.

Summarizing the above considerations, it is quite clear that the cross-calibration geometry can be optimized in relation to the number of photons reaching the pixels of the telescopes. The optimization procedure, however, is far from being trivial, and this is for the following reasons. First of all, the results above suggest that in terms of elevation angle and altitude, each pixel of the telescope has essentially its own optimal regime, and hence that the optimal calibration configuration cannot be achieved just as the simple superposition of the individual pixels optimal regimes. Second of all, the present work only gives part of the picture, namely that part corresponding to 


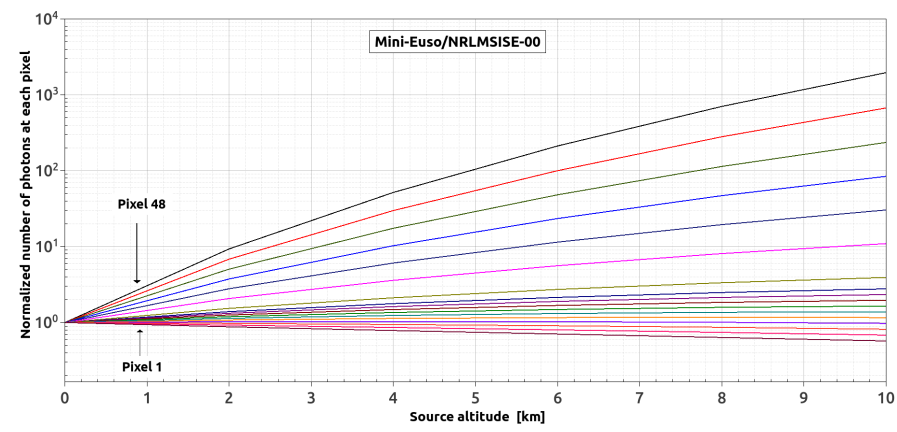

Figure 6: Normalized number of photons per pixel for Mini-EUSO as a function of source altitude for for $\delta=0^{\circ}, \mathrm{I}_{\text {beam }}=10^{11} \mathrm{~W} / \mathrm{m}^{2}$ and $6 \mathrm{~mm}$ beam diameter. The number of photons at each pixel is normalized to the corresponding value at sea-level.

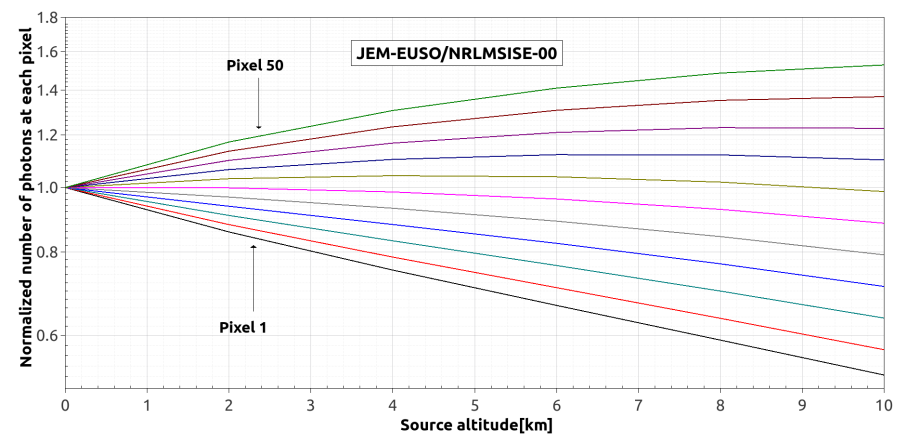

Figure 7: Normalized number of photons per pixel for Mini-EUSO as a function of source altitude for for $\delta=0^{\circ}, \mathrm{I}_{\text {beam }}=10^{11} \mathrm{~W} / \mathrm{m}^{2}$ and $6 \mathrm{~mm}$ beam diameter. The number of photons at each pixel is normalized to the corresponding value at sea-level.

geomagnetically quiescent midlatitude atmospheres, and the full optimization procedure should also take into account other effects such as aerosols, clouds, more active geomagnetic activity, etc. Finally, the optimization of the calibration procedure must also take into account the effects of the DAQ systems and of the associated triggering schemes, as well as the particular characteristics of the "beam reconstruction" methodology that will be used to validate the calibration procedure. Nevertheless, the present work gives a rather accurate overview of the issues that need to be solved in the future in order to develop a reliable ground-based cross-calibration procedure based on the indirect illumination of orbital telescopes.

\section{Conclusions}

The conclusions of the present work are quite straightforward. For the ground-based crosscalibration of orbital UV telescopes such as Mini-EUSO and JEM-EUSO, it is possible to use essentially the same laser sources that are presently used by the PAO and TA CLFs, and in the particular case of JEM-EUSO, a light-source system based on high-power LEDs is a possible alternative. Moreover, the calibration geometry is optimizable, but the development of a high- 
performance optimization procedure must necessarily rely on more than just the geometric aspects discussed here.

Under these circumstances, our future plans in this respect are to improve our GBSatCal simulation software to include in the light transport to the source other relevant effect such as humidity, aerosols and clouds, as well as to investigate the influence of the DAQ electronics and "beam reconstruction" methodology on the calibration optimization procedure.

Acknowledgment: This work was partially supported by Basic Science Interdisciplinary Research Projects of RIKEN and JSPS KAKENHI Grant (22340063, 23340081, and 24244042), by the Italian Ministry of Foreign Affairs, General Direction for the Cultural Promotion and Cooperation, by the 'Helmholtz Alliance for Astroparticle Physics HAP' funded by the Initiative and Networking Fund of the Helmholtz Association, Germany, and by Slovak Academy of Sciences MVTS JEM-EUSO as well as VEGA grant agency project 2/0076/13. Russia is supported by the Russian Foundation for Basic Research Grant No 13-02-12175-ofi-m. The Spanish Consortium involved in the JEM-EUSO Space Mission is funded by MICINN \& MINECO under the Space Program projects: AYA200906037-E/AYA, AYA-ESP2010-19082, AYA-ESP2011-29489-C03, AYA-ESP2012-39115-C03, AYA-ESP2013-47816C4, MINECO/FEDER-UNAH13-4E-2741, CSD2009-00064 (Consolider MULTIDARK) and by Comunidad de Madrid (CAM) under projects S2009/ESP-1496 \& S2013/ICE-2822.

\section{References}

[1] J. Abraham et al., Nuclear Instruments and Methods in Physics Research A 620 (2-3) (2010) 227-251.

[2] H. Kawai et al., Nuclear Physics B (Proc. Suppl) 175-176 (2008) 221-226.

[3] B. Fick et al., Journal of Instrumentation 1 (2006) P11003.

[4] S. Udo et al., Proc. of the 30th International Cosmic Ray Conference 5 (2008) 1021-1024 (ID 800).

[5] T. Ebisuzaki et al., Advances in Space Research 53(10) (2014) 1490-1505.

[6] P. Gorodetzky, T. Ebisuzaki, Workshop on laser solutions to remove space debris, APC, Paris, 27-28 April, 2015.

[7] B. A. Khrenov et al., European Physical Journal Web of Conferences 53 (2013) 9006.

[8] M. I. Panasyuk et al., Proceedings of the 34th International Cosmic Ray Conference (to be published) 2015.

[9] Y. Kawasaki et al., Proc. of the 32nd International Cosmic Ray Conference 3 (2011) 96-98 (ID 0472).

[10] T. Mernik et al., Proc. of the 33rd International Cosmic Ray Conference (2013), 55-58 (ID 0777).

[11] H. Adams Jr. et al., Astroparticle Physics 44 (2013) 76-90.

[12] LedEngin LZC-70U600 (http://www.ledengin.com/files/products/LZC/LZC-00U600.pdf).

[13] US Standard Atmosphere 1976, NASA Technical Memorandum TM-X-74335 (1976).

[14] J. M. Picone, A. Hedin, D. P. Drob, Journal of Geophysical Research 107 (A12) (2002) 1468-1484. 